\title{
Qual a influência da arquitetura muscular na funcionalidade de idosos? Uma revisão narrativa
}

\author{
What is the influence of muscle architecture on the functionality of the elderly? A narrative \\ review
}

\section{¿Cuál es la influencia de la arquitectura muscular en la funcionalidad de los adultos mayores? Una revisión narrativa}

\begin{abstract}
Klauber Dalcero Pompe ${ }^{\mathrm{I}}$ Eliane Celina Guadagnin ${ }^{\mathrm{II}}$, Patrícia Freitas dos Santos ${ }^{\mathrm{III}}$, Emmanuel Souza da Rocha, Rodrigo Rodrigues ${ }^{\mathrm{IV}}$
\end{abstract}

\begin{abstract}
Resumo
O envelhecimento do corpo acarreta alterações na arquitetura muscular, com consequente efeito deletério sobre a capacidade funcional e independência de idosos. Compreender como os parâmetros de arquitetura muscular interferem na funcionalidade do músculo esquelético, especialmente em idosos, é de fundamental importância para os profissionais da saúde que trabalham em processos de treinamento e reabilitação. O objetivo deste estudo é realizar uma revisão narrativa sobre os parâmetros e as adaptações na arquitetura muscular no envelhecimento e as implicações na funcionalidade de pessoas idosas. A arquitetura muscular afeta a funcionalidade do músculo esquelético, conforme a disposição das fibras musculares, em relação ao eixo de geração de força. A melhora na arquitetura muscular reflete-se em um melhor desempenho em tarefas funcionais e na independência dos idosos nas atividades de vida diárias. O treinamento de força é efetivo para minimizar os efeitos deletérios do envelhecimento na arquitetura muscular e reduzir os impactos negativos na capacidade funcional.
\end{abstract}

Palavras-chave: Arquitetura muscular; Músculo esquelético; Envelhecimento; Adaptação funcional

\begin{abstract}
The aging process causes negative changes in muscle architecture, with a consequent deleterious effect on the functional capacity and independence of the elderly. Understanding how muscular architecture parameters interfere with skeletal muscle functionality, especially in the elderly, is fundamentally important for health professionals who work in the training and rehabilitation processes, since it can contribute to the structuring of activities for this population. The present study aims to develop a narrative review of the parameters and adaptations in muscle architecture with aging and its implications for the functionality of the elderly. Improvements in the muscle architecture result in a better performance on functional tasks and the elderly's independence to perform their daily living activities. Strength training programs effectively minimize the deleterious effects of the muscle architecture aging process and reduce the negative impacts on functional capacity.
\end{abstract}

Keywords: Muscle architecture; Skeletal muscle; Elderly; Functionality

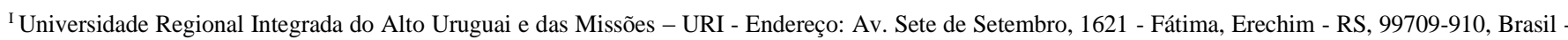
e-mail: kdpompeo@yahoo.com.br

II Filiação autor - e-mail: elianecguadagnin@ hotmail.com

III Filiação autor - e-mail: patidfreitas@ hotmail.com

IV Filiação autor - e-mail: emmarocha@gmail.com

v Filiação autor - e-mail: rodrigo.esef@gmail.com
} 


\section{Resumen}

El proceso de envejecimiento provoca cambios en la arquitectura muscular con el consecuente efecto deletéreo sobre la capacidad funcional y la independencia del adulto mayor. El comprender cómo los parámetros de la arquitectura muscular influyen en la funcionalidad del músculo esquelético, especialmente en el adulto mayor, es de fundamental importancia para los profesionales de la salud que trabajan en procesos de entrenamiento y rehabilitación. El objetivo de este estudio es realizar una revisión narrativa sobre los parámetros y las adaptaciones de la arquitectura muscular en el envejecimiento, así como también conocer las implicaciones que posee sobre la funcionalidad en los adultos mayores. La arquitectura muscular afecta la funcionalidad del músculo esquelético, según la disposición de las fibras musculares en relación con el eje de generación de fuerza. La mejora en la arquitectura muscular se refleja en un mejor desempeño de las tareas funcionales y en la independencia del anciano en las actividades de la vida diaria. El entrenamiento de fuerza es eficaz para minimizar los efectos nocivos del envejecimiento en la arquitectura muscular y reducir los impactos negativos sobre la capacidad funcional.

Palabras clave: Arquitectura muscular; Músculo esquelético; Envejecimiento; Adaptación funcional

\section{Introdução}

O músculo esquelético é o tecido mais abundante no corpo humano, o qual representa 40-45\% do peso corporal total (NORDIN; FRANKEL, 2012). Esta estrutura realiza ativamente tarefas estáticas e dinâmicas, confere força e proteção ao esqueleto, mantém a postura, e faz com que os ossos se movam nas articulações (NORDIN; FRANKEL, 2012). Os músculos esqueléticos apresentam-se em uma variedade de formas e tamanhos, sendo que, pela disposição das fibras musculares, podem ser classificados como paralelos ou oblíquos (LIPPERT, 2018). Esse arranjo ou organização macroscópica das fibras musculares é definido como arquitetura muscular (GANS; GAUNT, 1991; LIEBER; FRIDÉN, 2000).

A arquitetura muscular considera o arranjo interno das fibras musculares em relação ao eixo de geração de força do músculo (LIEBER, 1992), e influencia a capacidade de produção de força muscular, assim como a capacidade de excursão (amplitude de trabalho) do músculo (LIEBER; FRIDÉN, 2000). O processo de envelhecimento e o treinamento físico possuem a capacidade de modificar a arquitetura muscular (KUBO et al., 2003; FRANCHI et al., 2014). Além disso, observa-se a existência de uma relação entre a arquitetura muscular e a capacidade funcional do ser humano na performance esportiva (KUMAGAI et al., 2000) e na execução de atividades da vida diária (GUADAGNIN et al., 2019),

Alguns estudos mostram que o processo de envelhecimento está associado a alterações na arquitetura muscular (YOUNG; STOKES; CROWE, 1984; MEN et al., 1985; NARICI; MAGANARIS, 2006; CLARK et al., 2014). De forma similar, o desempenho em tarefas funcionais também é afetado com o avanço da idade (FERRUCCI et al., 2016; LANDI et al., 2017). A relação entre a arquitetura 
muscular e o desempenho em tarefas funcionais, em idosos, vem sendo explorada em alguns estudos de associação (WILHELM et al., 2014; SELVA RAJ; BIRD; SHIELD, 2017; GUADAGNIN et al., 2019). Algumas revisões existentes sobre o assunto tratam sobre o comportamento da arquitetura muscular com o envelhecimento (BAPTISTA; VAZ, 2009; PERKISAS et al., 2016), e como isso está associado à produção de força e ao uso aumentado e uso reduzido. No entanto, estudos de revisão que abordem a associação entre a arquitetura muscular e a funcionalidade de idosos ainda precisam ser mais explorados.

Compreender os conceitos de arquitetura muscular, as relações existentes entre as características morfológicas dos músculos esqueléticos e a funcionalidade do ser humano é fundamental para os profissionais da área da saúde, durante a elaboração e aplicação de um programa de treinamento ou reabilitação, assim como, na compreensão das adaptações funcionais decorrentes do processo natural de envelhecimento. Considerando o aumento da longevidade e da expectativa de vida dos indivíduos, faz-se necessário trazer à luz evidências encontradas na literatura quanto às modificações decorrentes do processo de envelhecimento na arquitetura muscular, além da associação entre a arquitetura muscular e o desempenho em tarefas funcionais em idosos.

Com base no exposto, este estudo tem como objetivo desenvolver uma revisão narrativa de literatura sobre os conceitos teóricos dos parâmetros de arquitetura muscular, as alterações observadas nesses parâmetros associadas ao processo de envelhecimento e a relação entre arquitetura muscular e funcionalidade de idosos.

\section{Metodologia}

Este estudo se caracteriza como uma revisão narrativa desenvolvida a partir da análise de artigos originais, artigos de revisão da literatura e livros didáticos publicados sobre a temática envelhecimento, arquitetura muscular e funcionalidade. Para a realização das buscas, não houve qualquer restrição quanto a data de publicação da literatura utilizada. Utilizou-se os seguintes termos (combinados ou não) para a realização de buscas: "muscle architecture, elderly, older aging, functionality strength training, resistance training, envelhecimento, idosos, treinamento de força, funcionalidade, adaptação funcional e arquitetura muscular". Os artigos científicos foram buscados nas bases de dados Pubmed, Scopus, Cochrane e Scielo. Foram procurados materiais que abordassem os conceitos teóricos de arquitetura muscular, o efeito do envelhecimento e do treinamento sobre esses parâmetros e estudos que analisassem a associação entre a arquitetura muscular e a funcionalidade de idosos. Nos estudos de revisão narrativa, não é necessário informar a metodologia utilizada para a busca das referências, nem os critérios utilizados 
na avaliação e seleção dos trabalhos consultados (BERNARDO; NOBRE; JATENE, 2004). Nos estudos do tipo revisões narrativas ou estudo do tipo estado da arte, são avaliadas as produções científicas em determinada área do conhecimento, para fornecer o estado da arte sobre uma temática específica (NORONHA; FERREIRA, 2000). Os artigos selecionados que abordam as relações existentes entre a arquitetura muscular e a funcionalidade, e os efeitos do treinamento para idosos estão apresentados na Tabela 1.

Tabela 1 - Estudos que avaliaram a relação entre a arquitetura muscular e a funcionalidade e treinamento e funcionalidade em idosos

\begin{tabular}{|c|c|c|}
\hline Autores e ano & Tópico(s) avaliado(s) & Resultado(s) \\
\hline \multicolumn{3}{|c|}{ Relação entre arquitetura muscular e funcionalidade } \\
\hline Clark et al. 2014 & $\begin{array}{l}\text { - Correlação entre área de secção } \\
\text { transversa dos músculos da coxa e } \\
\text { velocidade de caminhada em } \\
\text { homens e mulheres (teste de } 400 \mathrm{~m} \text { ) }\end{array}$ & $\begin{array}{l}\text { - Correlação positiva significativa para } \\
\text { os homens, mas não para as mulheres }\end{array}$ \\
\hline Rech et al. 2014 & $\begin{array}{l}\text { - Correlação de espessura do } \\
\text { quadríceps e teste de sentar e } \\
\text { levantar 30s e de velocidade } \\
\text { preferida de marcha }(6 \mathrm{~m})\end{array}$ & - Não houve correlação significativa \\
\hline Wilhelm et al. 2014 & $\begin{array}{l}\text { - Correlação de espessura de } \\
\text { quadríceps com teste de sentar e } \\
\text { levantar 30s }\end{array}$ & $\begin{array}{l}\text { - Correlação positiva significativa da } \\
\text { espessura com o número de repetições } \\
\text { em 30s }\end{array}$ \\
\hline Lopez et al. 2017 & $\begin{array}{l}\text { - Correlação entre espessura do } \\
\text { quadríceps e teste de sentar e } \\
\text { levantar 30s }\end{array}$ & $\begin{array}{l}\text { - Correlação positiva significativa entre } \\
\text { espessura e o número de repetições em } \\
\text { 30s }\end{array}$ \\
\hline Selva et al. 2017 & $\begin{array}{l}\text { - Correlação entre espessura } \\
\text { muscular do gastrocnêmio medial, } \\
\text { vasto lateral, e vasto intermédio + } \\
\text { reto femoral e tempo no teste de } \\
\text { caminhada de } 6 \mathrm{~m} \\
\text { - Correlação entre ângulo de penação } \\
\text { e comprimento de fascículo de vasto } \\
\text { lateral e gastrocnêmio medial e } \\
\text { tempo no teste de caminhada de } 6 \mathrm{~m}\end{array}$ & $\begin{array}{l}\text { - Correlação negativa significativa entre } \\
\text { a espessura e o tempo para realizar o } \\
\text { teste } \\
\text { - Sem correlação significativa }\end{array}$ \\
\hline $\begin{array}{c}\text { Guadagnin et al. } \\
2019\end{array}$ & $\begin{array}{l}\text { - Correlação entre espessura do vasto } \\
\text { lateral e velocidade preferida e } \\
\text { máxima da marcha } \\
\text { - Correlação entre velocidade } \\
\text { máxima da marcha e ângulo de } \\
\text { penação e comprimento de fascículo } \\
\text { do tibial anterior } \\
\text { - Correlação entre estrutura } \\
\text { velocidade de marcha preferida e } \\
\text { máxima e estrutura do bíceps }\end{array}$ & $\begin{array}{l}\text { - Correlação positiva significativa entre } \\
\text { espessura do vasto lateral e velocidade } \\
\text { preferida e máxima da marcha } \\
\text { - Correlação positiva significativa da } \\
\text { velocidade com ângulo de penação e } \\
\text { negativa com comprimento de fascículo } \\
\text { - Sem correlação significativa }\end{array}$ \\
\hline
\end{tabular}




\begin{tabular}{|c|c|c|}
\hline & $\begin{array}{l}\text { femoral, do gastrocnêmio medial e } \\
\text { do reto femoral } \\
\text { - Correlação entre velocidade } \\
\text { preferida e máxima de marcha com } \\
\text { transposição de obstáculo } \\
\text { - Correlação entre espessura do tibial } \\
\text { anterior e distância vertical entre pé } \\
\text { e obstáculo }\end{array}$ & $\begin{array}{l}\text { - Sem correlação significativa } \\
\text { - Correlação positiva significativa entre } \\
\text { espessura do tibial anterior e distância } \\
\text { vertical pé-obstáculo }\end{array}$ \\
\hline Komforti et al. 2020 & $\begin{array}{l}\text { - Correlação entre área de secção } \\
\text { transversa de reto femoral, vasto } \\
\text { lateral e gastrocnêmio lateral com } \\
\text { velocidade máxima da marcha }(4 \mathrm{~m})\end{array}$ & $\begin{array}{l}\text { - Correlação positiva significativa entre } \\
\text { área de secção transversa e velocidade } \\
\text { máxima }\end{array}$ \\
\hline $\begin{array}{l}\text { Mateos-Angulo et al. } \\
\qquad 2020\end{array}$ & $\begin{array}{l}\text { - Correlação entre espessura } \\
\text { muscular reto femoral e teste de } \\
\text { sentar e levantar de cinco repetições }\end{array}$ & $\begin{array}{l}\text { - Correlação negativa significativa entre } \\
\text { espessura e tempo para realizar cinco } \\
\text { repetições }\end{array}$ \\
\hline \multicolumn{3}{|c|}{ Efeitos do treinamento de força sobre a funcionalidade } \\
\hline Cadore et al. 2014 & $\begin{array}{l}\text { - Treinamento de força concêntrico e } \\
\text { excêntrico e espessura muscular do } \\
\text { vasto lateral }\end{array}$ & $\begin{array}{l}\text { - Ambos os tipos de treinamento } \\
\text { melhoraram a espessura muscular do } \\
\text { vasto lateral }\end{array}$ \\
\hline Váczi et al. 2014 & $\begin{array}{l}\text { - Treinamento de força convencional } \\
\mathrm{x} \text { excêntrico e espessura muscular }\end{array}$ & $\begin{array}{l}\text { - Ambos os grupos aumentaram a } \\
\text { espessura muscular }\end{array}$ \\
\hline Dias et al. 2015 & $\begin{array}{l}\text { - Treinamento de força excêntrico } x \\
\text { convencional 1RM de extensão de } \\
\text { joelho }\end{array}$ & $\begin{array}{l}\text { - Ambos os grupos melhoraram no } \\
\text { parâmetro força }\end{array}$ \\
\hline \multirow[t]{3}{*}{ Franchi et al. 2015} & $\begin{array}{l}\text { - Treinamento de força concêntrico x } \\
\text { excêntrico e espessura muscular }\end{array}$ & $\begin{array}{l}\text { - Ambos os grupos aumentaram a } \\
\text { espessura muscular sem diferença entre } \\
\text { os grupos }\end{array}$ \\
\hline & - Comprimento do fascículo & $\begin{array}{l}\text { - Ambos os grupos aumentaram o } \\
\text { comprimento do fascículo sem } \\
\text { diferença entre os grupos }\end{array}$ \\
\hline & - Ângulo de penação & $\begin{array}{l}\text { - Ambos os grupos melhoraram ângulo } \\
\text { de penação }\end{array}$ \\
\hline Timmins et al. 2016 & $\begin{array}{l}\text { - Treinamento de força concêntrico } \mathrm{x} \\
\text { excêntrico e comprimento do } \\
\text { fascículo }\end{array}$ & $\begin{array}{l}\text { - Ambos os grupos aumentaram o } \\
\text { comprimento do fascículo }\end{array}$ \\
\hline Lastayo et al. 2017 & $\begin{array}{l}\text { - Treinamento de força convencional } \\
\mathrm{x} \text { excêntrico e espessura muscular, } \\
\text { comprimento do fascículo e ângulo } \\
\text { de penação }\end{array}$ & $\begin{array}{l}\text { - Ambos os grupos obtiveram aumento } \\
\text { nos parâmetros de arquitetura muscular }\end{array}$ \\
\hline
\end{tabular}

Fonte: autoria 


\section{Discussão}

\subsection{Morfologia muscular}

Quanto à disposição das fibras musculares em relação ao seu eixo longitudinal, os músculos esqueléticos podem ser classificados como paralelos ou oblíquos (LIPPERT, 2011; 2018). Os músculos com fibras paralelas tendem a apresentar fibras mais longas e, portanto, com maior potencial de amplitude de movimento (LIPPERT, 2011). Nesse sentido, músculos com fibras mais longas possuem uma velocidade de encurtamento mais elevada, quando comparados aos músculos com fibras mais curtas (CARPES et al., 2011). Quanto maior a quantidade de sarcômeros em série, mais longa é a miofibrila (NORDIN; FRANKEL, 2012), dessa forma, nos músculos com disposição das fibras em paralelo, predomina o chamado efeito em série. Os músculos com a disposição das fibras em paralelo podem ser subdivididos, ainda, em: retos, fusiformes, planos ou triangulares (LIPPERT, 2011).

Por outro lado, os músculos com fibras oblíquas tendem a ter fibras mais curtas e mais numerosas por área, fazendo com que os músculos oblíquos tenham maior potencial de força, mas com menor amplitude de movimento (LIPPERT, 2011). Os músculos com a disposição das fibras oblíquas apresentam inserção em ângulo oblíquo em relação ao seu tendão, e podem ser classificados como: semipeniforme (unipenado), peniforme (bipenado) ou multipeniforme (multipenado) (NEUMANN, 2010; LIPPERT, 2011) (Figura 1).

Figura 1. Classificação quanto à arquitetura muscular. (A) Músculo semipeniforme (unipenado), tendo como exemplo o músculo Bíceps femoral cabeça longa; (B) Músculo peniforme (bipenado), tendo como exemplo o músculo reto femoral; (C) Músculo multipeniforme (multipenado), tendo como exemplo o músculo deltoide.

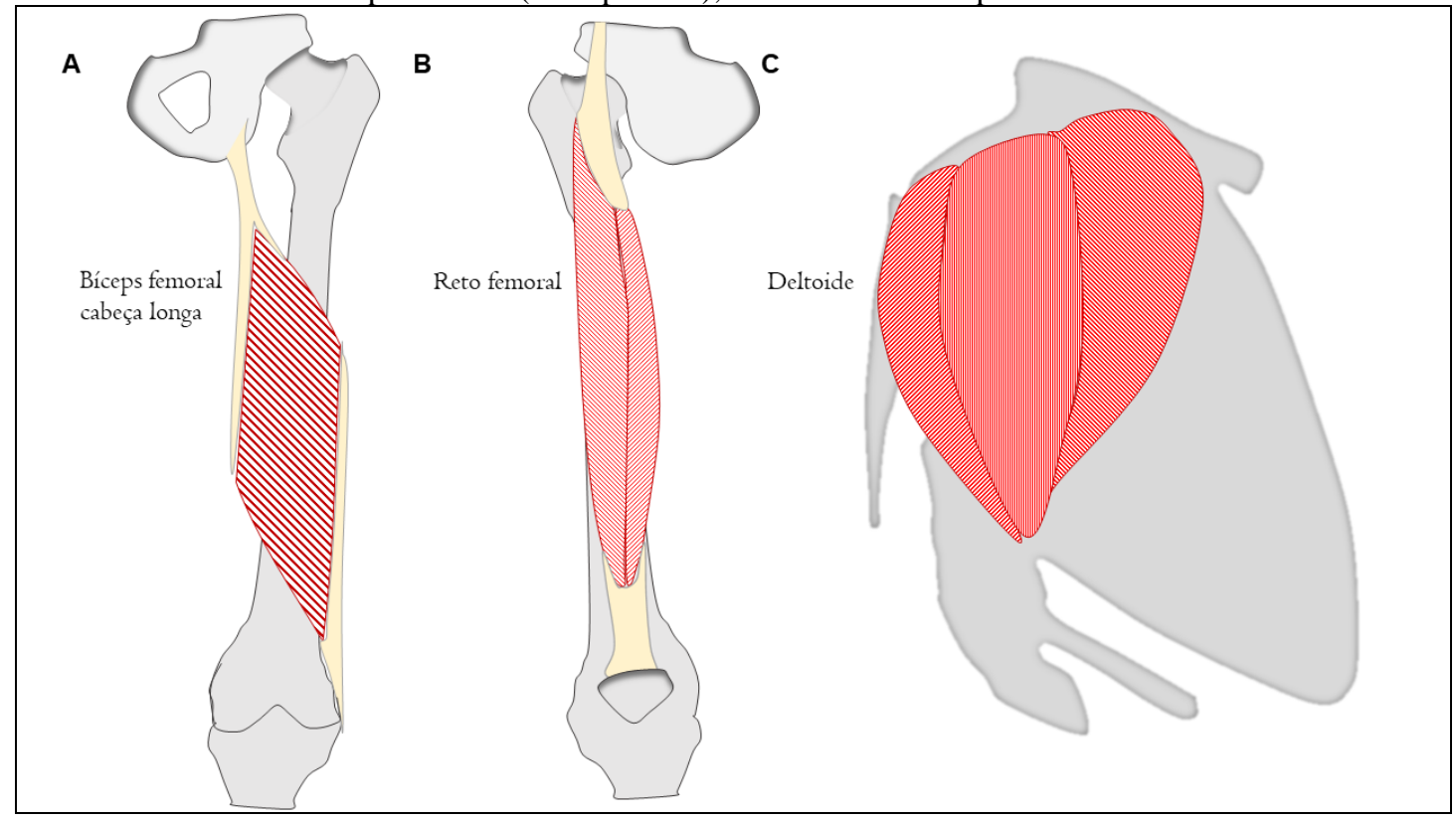

Fonte: os autores, imagem desenvolvida no software Power Point. 
A penação das fibras musculares acarreta maior área de secção transversa fisiológica, fazendo com que mais fibras atuem em paralelo para a produção da força (CARPES et al., 2011), predominando, dessa forma, o chamado efeito em paralelo nos músculos oblíquos. Resumidamente, pode-se dizer que músculos com fibras curtas e maior área transversa são projetados para produzir força, enquanto que músculos com fibras longas são projetados para excursão e velocidade (NORDIN; FRANKEL, 2012).

\subsection{Parâmetros de Arquitetura Muscular}

Inicialmente, julga-se necessário definir conceitualmente os principais parâmetros de arquitetura muscular comumente avaliados:

Ângulo de penação: ângulo de orientação entre as fibras musculares e o tendão (NEUMANN, 2010). O ângulo de penação é calculado como o ângulo entre o fascículo e a aponeurose profunda (BARONI et al., 2013) (Figura 2). Quanto maior o ângulo de penação, menor a força transferida pela fibra para o tendão (KAWAKAMI, 2005);

Comprimento de fascículo: comprimento fascicular entre as aponeuroses superficial e profunda (Figura 2). Quanto maior o comprimento do fascículo, maior a velocidade de encurtamento do músculo (KUMAGAI et al., 2000; LIEBER; FRIDÉN, 2000);

Espessura muscular: distância entre a aponeurose superficial e a aponeurose profunda (Figura 2).

Figura 2. Na imagem à esquerda, uma ultrassonografia longitudinal, estão apresentados os seguintes parâmetros de arquitetura muscular: ângulo de penação (AP), comprimento de fascículo (CF) e espessura muscular (EM); na imagem à direita, uma ultrassonografia transversal, está desenhada manualmente a área de secção transversa anatômica (ASTA). Imagens obtidas dos músculos vasto lateral (imagem à esquerda) e reto femoral (imagem à direita), a 50\% da distância entre o trocânter maior e o côndilo lateral do fêmur, com equipamento de ultrassonografia (Logiq P6, GE Healthcare, Waukesha, Washington, EUA) e sonda matricial de arranjo linear (60mm, ML6-15, 5-15Mhz - GE Healthcare, Waukesha, Washington, EUA).

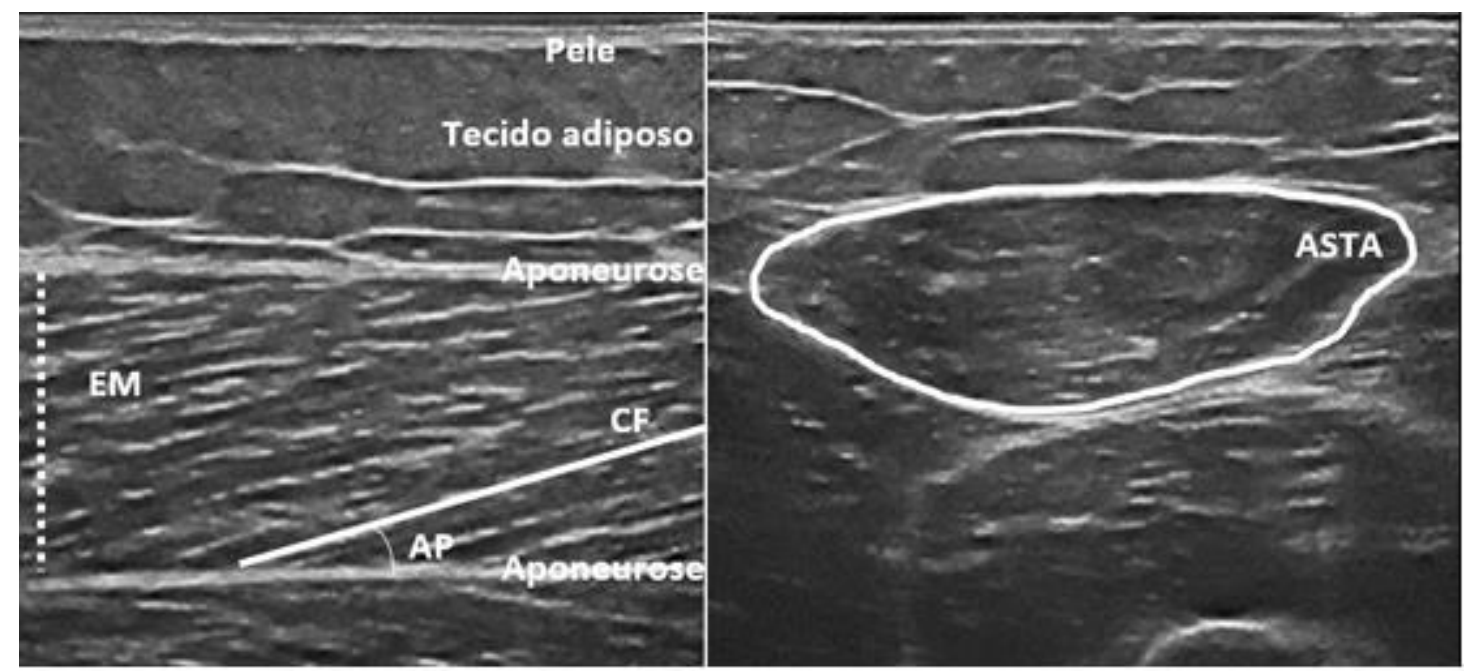

Fonte: os autores, imagem desenvolvida no software Power Point 
Área de secção transversa anatômica: corte transversal perpendicular à direção das fibras musculares, sem considerar sua penação (Figura 2 e 3);

Área de secção transversa fisiológica: considera o corte transversal perpendicular à orientação das fibras musculares (CARPES et al., 2011) e reflete a quantidade de proteínas ativas disponíveis à geração de força de contração (NEUMANN, 2010). Representa o somatório das áreas de secção transversa de todas as fibras musculares presentes em um músculo (LIEBER; FRIDÉN, 2000). Cabe destacar que a área de secção transversa fisiológica é o único parâmetro de arquitetura muscular diretamente proporcional à força máxima gerada pelo músculo (LIEBER; FRIDÉN, 2000) (Figura 3).

Figura 3. Representação esquemática de um músculo com fibras paralelas (A) e um músculo com fibras oblíquas/ unipenado (B) e suas respectivas áreas de secção transversa anatômica (ASTA, linha contínua) e área de secção transversa fisiológica (linha pontilhada). Note que a ASTA (linha contínua) não se modifica, independente do arranjo das fibras musculares.

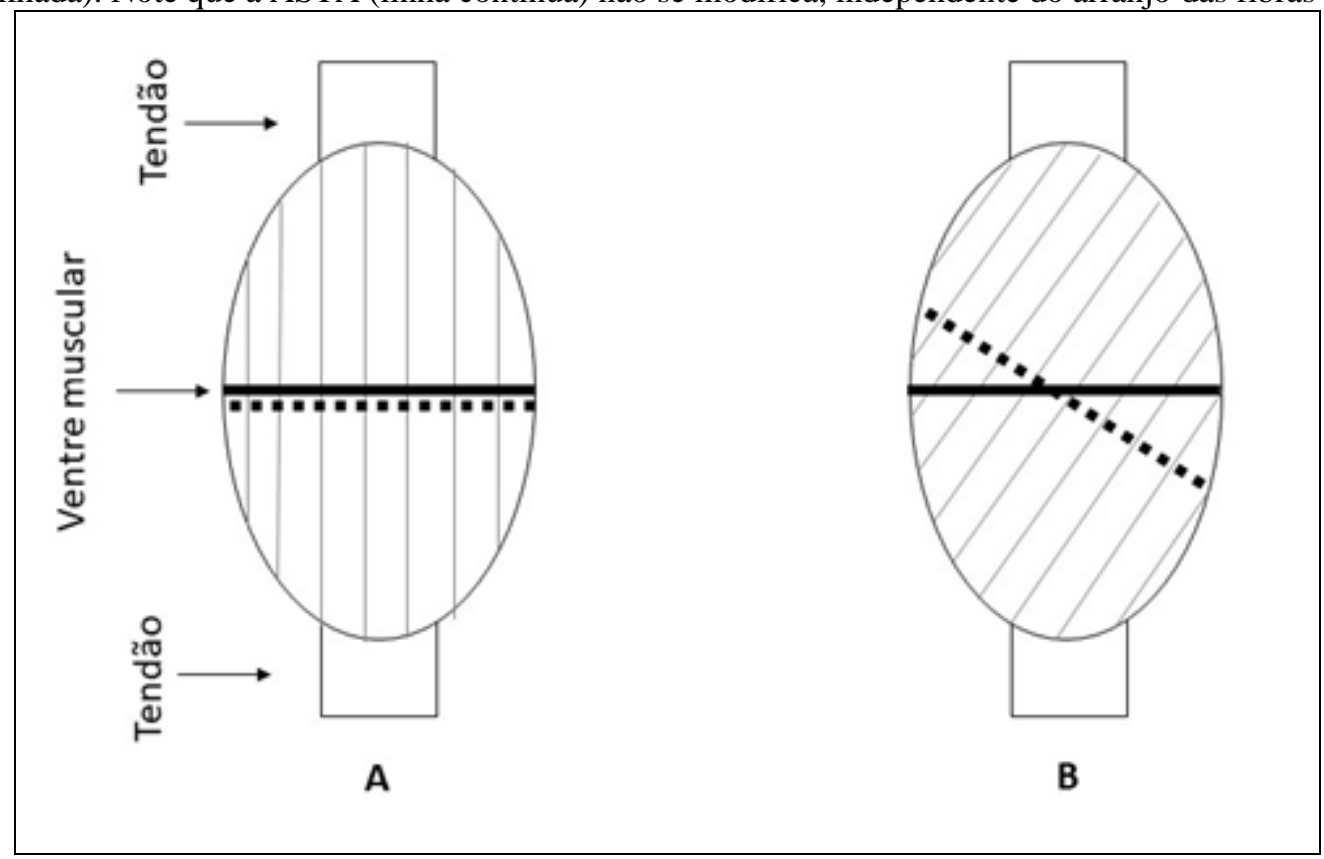

Fonte: os autores, imagem desenvolvida no software Power Point.

Cabe destacar que os parâmetros de arquitetura muscular não ficam inalterados, por exemplo, durante um movimento articular, o ângulo de penação e o comprimento do fascículo se alteram em função da posição articular. Para exemplificar, o músculo vasto lateral reduz em $18 \%$ o comprimento do fascículo e aumenta em $21 \%$ o ângulo de penação durante a extensão passiva do joelho $\left(100^{\circ}-20^{\circ}\right.$, sendo $0^{\circ}$ extensão completa) (FUKUNAGA et al., 1997). Além disso, observam-se diferenças na arquitetura muscular ao longo do comprimento do músculo (BLAZEVICH; GILL; ZHOU, 2006). Por exemplo, no músculo reto femoral, o maior ângulo de penação é encontrado na região proximal, enquanto que, no músculo vasto medial, o maior ângulo de penação é encontrado na região distal (BLAZEVICH; GILL; ZHOU, 2006). 
Durante a transição da situação em repouso (músculo relaxado), para uma situação em contração voluntária máxima isométrica, também ocorrem mudanças na arquitetura muscular. Utilizando-se novamente o músculo vasto lateral como exemplo, ocorre uma redução de $24 \%$ no comprimento do fascículo e um aumento de $47 \%$ no ângulo de penação durante a contração voluntária máxima isométrica. Já as contrações voluntárias dinâmicas ocasionam diferentes variações na arquitetura muscular. Durante uma contração concêntrica, ocorre um aumento no ângulo de penação e uma redução no comprimento do fascículo (FRANCHI et al., 2014). Nas contrações excêntricas, ocorre um aumento no comprimento do fascículo, enquanto que o ângulo de penação não se altera significativamente (FRANCHI et al., 2014).

Também, é possível estabelecer interrelações entre os parâmetros de arquitetura muscular. Já foi previamente observada, na literatura, uma correlação significativa existente entre o ângulo de penação e a espessura muscular, com evidências de que a hipertrofia muscular é acompanhada de um incremento no ângulo de penação (KAWAKAMI; ABE; FUKUNAGA, 1993). Ao se comparar dois músculos com o mesmo volume muscular, aquele músculo que apresentar maior ângulo de penação, ou seja, maior ângulo de inserção em relação ao tendão, irá apresentar maior área de secção transversa fisiológica e, consequentemente, maior capacidade de produção de força (BLAZEVICH; SHARP, 2005).

Importante levar em consideração que a arquitetura muscular afeta a funcionalidade do músculo esquelético, como a capacidade de produção de força e velocidade de encurtamento (LIEBER; FRIDÉN, 2000) e que os parâmetros de arquitetura também adaptam-se ao uso aumentado (treinamento) (FRANCHI et al., 2014), ao uso reduzido (destreino) (CORREA et al., 2016) e ao processo de envelhecimento (NARICI et al., 2003), aspecto que será abordado a seguir.

\subsection{Arquitetura muscular e envelhecimento}

O processo de envelhecimento biológico é caracterizado por diferentes alterações na estrutura muscular. Essas mudanças impactam de forma negativa as características de função muscular, como a produção de força (YOUNG; STOKES; CROWE, 1984; MEN et al., 1985), e também, de forma geral, a funcionalidade do corpo dos idosos, uma vez que os parâmetros musculares apresentam relação com o desempenho em tarefas funcionais, como a velocidade de marcha e o sentar-e-levantar (CLARK et al., 2014; WILHELM et al., 2014; LOPEZ et al., 2017; SELVA RAJ; BIRD; SHIELD, 2017; GUADAGNIN et al., 2019; MATEOS-ANGULO; GALÁN-MERCANT; CUESTA-VARGAS, 2020; KOMFORTI et al., 2020). Apesar de essas características serem inerentes ao processo, o exercício físico tem um grande poder de amenizar e de reverter perdas na estrutura muscular (NILWIK et al., 2013), visto que indivíduos 
classificados como fisicamente ativos apresentam uma atenuação nas alterações destes parâmetros ao longo do processo de envelhecimento.

De forma geral, o que se observa é uma perda de massa muscular, a qual está associada a uma perda do número de fibras musculares (cerca de 39\% dos 20 aos 80 anos de idade) (LEXELL; TAYLOR; SJÖSTRÖM, 1988), bem como do tamanho das mesmas, especialmente das fibras do tipo II (de contração rápida), devido à morte dos motoneurônios que as inervam, levando a uma menor proporção de fibras do tipo II em idosos (TAYLOR; SJÖSTRÖM, 1988; NARICI; MAGANARIS, 2006; CRUZJENTOFT et al., 2010; LEXELL; NILWIK et al., 2013).

Como parte da perda de massa muscular, são vistas também reduções no número de sarcômeros em série e em paralelo, ocorrendo, portanto, uma redução no comprimento de fascículo, no ângulo de penação, na espessura muscular e na área de secção transversa anatômica. Comparações entre jovens e idosos apontam que o comprimento do fascículo é cerca de $15 \%$ menor nos idosos para o músculo vasto lateral (WU et al., 2016) e de 10,2\% para o gastrocnêmio medial (NARICI et al., 2003), além de também os idosos apresentarem reduções no ângulo de penação (NARICI et al., 2003; MORSE et al., 2005). Por fim, a espessura muscular também é menor em idosos (WATANABE et al., 2018), assim como a área de secção transversa muscular, cujas diferenças variam de cerca de $14 \%$ a $40 \%$, dependendo do estudo (YOUNG; STOKES; CROWE, 1984; MEN et al., 1985; MORSE et al., 2005; NILWIK et al., 2013; WATANABE et al., 2018).

\subsection{Arquitetura muscular e funcionalidade em idosos}

Considerando que as adaptações neuromusculares relacionadas ao envelhecimento levam o indivíduo a ter menor massa muscular e menor comprimento de fascículo, a produção de força e de velocidade podem também ser afetadas, tendo consequências sobre a funcionalidade, aspecto que mantém o idoso independente para a realização de suas atividades de vida diária. Nesse sentido, vários estudos têm investigado a associação entre a estrutura muscular e diferentes tarefas funcionais (CLARK et al., 2014; RECH et al., 2014; WILHELM et al., 2014; LOPEZ et al., 2017; SELVA RAJ; BIRD; SHIELD, 2017; GUADAGNIN et al., 2019; KOMFORTI et al., 2020; MATEOS-ANGULO; GALÁNMERCANT; CUESTA-VARGAS, 2020).

Uma das tarefas funcionais comumente avaliadas é o sentar e levantar de uma cadeira, que pode ser avaliada pelo número de vezes que o indivíduo realiza o movimento em determinado tempo ou pelo tempo que o indivíduo gasta para realizar um determinado número de repetições. Nessa tarefa, foi observado que homens idosos que realizaram mais repetições em 30 segundos também apresentavam 
maior espessura muscular total do quadríceps (WILHELM et al., 2014; LOPEZ et al., 2017), o que não ocorreu para mulheres idosas (RECH et al., 2014). Considerando o tempo para desempenhar cinco repetições, foi observado que idosos de ambos os sexos que realizaram a tarefa em menor tempo, apresentaram maior espessura muscular do reto femoral (MATEOS-ANGULO; GALÁN-MERCANT; CUESTA-VARGAS, 2020). Os demais parâmetros de arquitetura muscular e demais músculos de membros inferiores parecem não ter sido investigados ainda, não sendo possível afirmar se eles estão associados com o desempenho nessa tarefa.

Outra tarefa funcional que tem sido investigada é a marcha, em que a velocidade foi o aspecto mais abordado. Nesse sentido, observou-se que maiores áreas de secção transversa dos músculos da coxa estiveram associadas a maiores velocidades no teste de caminhada de 400 metros para homens idosos, mas não para mulheres (CLARK et al., 2014). Ainda, a espessura do quadríceps também não apresentou relação com a velocidade preferida de marcha em seis metros em mulheres idosas (RECH et al., 2014). Considerando idosos de ambos os sexos, maiores velocidades de marcha (preferida e máxima) foram associadas a maiores espessuras do vasto lateral (GUADAGNIN et al., 2019). A velocidade máxima de marcha esteve ainda associada a um maior ângulo de penação e a um menor comprimento do fascículo do tibial anterior (GUADAGNIN et al., 2019). Porém, nem a velocidade preferida, nem a máxima de marcha estiveram associadas com a estrutura do bíceps femoral, do gastrocnêmio medial e do reto femoral (GUADAGNIN et al., 2019).

Por outro lado, foi observado que idosos de ambos os sexos, com maiores áreas de secção transversa dos músculos vasto lateral, reto femoral e gastrocnêmio (lateral e medial), apresentavam maior velocidade máxima de marcha em uma distância de quatro metros (KOMFORTI et al., 2020). Isso se confirmou em outro estudo, em que um menor tempo no teste de caminhada rápida de 6 metros foi associado com maiores espessuras dos músculos vasto lateral, gastrocnêmio medial, e uma combinação do somatório da espessura do reto femoral e vasto intermédio. Entretanto, o ângulo de penação e o comprimento de fascículo dos músculos vasto lateral e do gastrocnêmio medial não apresentaram associação significativa no teste de caminhada rápida de 6 metros (SELVA RAJ; BIRD; SHIELD, 2017).

Quando se trata da locomoção com transposição de obstáculo, um estudo que investigou o papel da estrutura muscular de diferentes músculos dos membros inferiores observou que uma maior espessura do tibial anterior parece estar associada com um menor risco de tropeços para o membro de abordagem ao obstáculo, representado por uma maior distância vertical entre o pé e o obstáculo no momento da transposição (GUADAGNIN et al., 2019). A velocidade de marcha com obstáculo não apresentou associação com a estrutura muscular (GUADAGNIN et al., 2019). 
3.5 Efeitos do treino de força para idosos

Durante o processo de envelhecimento, ocorrem perdas (em série e em paralelo) na arquitetura muscular, e a recuperação dessas perdas. Mesmo o retardamento delas durante o processo natural de envelhecimento é essencial. $\mathrm{O}$ treinamento de força tem se mostrado muito eficaz nesse sentido, sendo essa prática muito recomendada (REEVES et al., 2009; CADORE et al., 2014; VÁCZI et al., 2014; DIAS et al., 2015; LASTAYO et al., 2017).

O treinamento puramente concêntrico tem como resultado principal um aumento no ângulo de penação das fibras musculares (relacionado ao aumento dos sarcômeros - ou das miofibrilas - em paralelo), gerando também um aumento da espessura muscular e um aumento na área de secção transversa (CADORE et al., 2014; FRANCHI et al., 2015; WISDOM; DELP; KUHL, 2015; TIMMINS et al., 2016). Já o treino excêntrico, além do efeito em paralelo, gera aumento no comprimento dos fascículos musculares (decorrente do aumento do número de sarcômeros em série em cada miofibrila - ou hipertrofia em série) (REEVES et al., 2009; FRANCHI et al., 2015; TIMMINS et al., 2016).

O princípio da especificidade do treinamento esportivo indica que adaptações específicas devem ser obtidas após diferentes tipos de treinamento de força, e que as diferentes sobrecargas mecânicas devem gerar diferentes adaptações estruturais e, consequentemente, diferentes ganhos na capacidade de geração de força muscular. Entretanto, parece não existir diferença significativa na capacidade de produção de força, quando comparados os efeitos dos dois tipos de treinamento (ex: concêntrico e excêntrico) (VÁCZI et al., 2014; DIAS et al., 2015; LASTAYO et al., 2017), apesar dos diferentes efeitos e das diferentes sobrecargas mecânicas sobre a unidade músculo-tendão gerados pelos diferentes tipos de contração.

Diante das adaptações citadas na arquitetura muscular, a partir do treinamento de força (REEVES et al., 2009; CADORE et al., 2014; VÁCZI et al., 2014; DIAS et al., 2015; LASTAYO et al., 2017) e a relação entre estes parâmetros e a capacidade funcional em idosos (GUADAGNIN et al., 2019), o aumento na espessura do reto femoral pode beneficiar no desempenho do tempo para realizar cinco repetições no teste de sentar e levantar (MATEOS-ANGULO; GALÁN-MERCANT; CUESTAVARGAS, 2020). Ainda, o aumento da área de secção transversa do quadríceps, especialmente no vasto lateral e em músculos da perna, como gastrocnêmio medial e lateral, pode beneficiar na velocidade máxima de idosos (SELVA RAJ; BIRD; SHIELD, 2017; KOMFORTI et al., 2020). Por fim, um aumento na espessura do tibial anterior pode ser importante na transposição de obstáculos, reduzindo o risco de tropeços e quedas em idosos (GUADAGNIN et al., 2019). 


\section{Conclusão}

A arquitetura muscular afeta significativamente a funcionalidade do musculoesquelético, afetando a produção de força ou de velocidade deste músculo, dependendo do arranjo de suas fibras. O processo de envelhecimento impacta de maneira negativa a arquitetura muscular, prejudicando a execução de tarefas funcionais por idosos. O exercício físico, especialmente o treinamento de força, é uma forma de reverter os efeitos deletérios do envelhecimento na arquitetura muscular, o que pode gerar benefícios na realização de atividades de vida diária. Assim, identificar como esses parâmetros estruturais influenciam no funcionamento do músculo esquelético e na funcionalidade de idosos é de primordial importância aos profissionais que trabalham com o movimento humano, a fim de melhorar o desempenho funcional e a qualidade de vida dessa população, por meio da estruturação de programas de intervenção adequados.

\section{Referências}

BAPTISTA, R.R ; VAZ, M.A. Arquitetura muscular e envelhecimento: adaptação funcional e aspectos clínicos; revisão da literatura. Fisioterapia e Pesquisa, v. 16, n. 4, p. 368-373, dez. 2009.

BARONI, B.M. et al. Time course of neuromuscular adaptations to knee extensor eccentric training. International Journal of Sports Medicine, v. 34, n. 10, p. 904-911, mar. 2013.

BERNARDO, W.M; NOBRE, M.R.C; JATENE, F.B. A Prática Clínica Baseada em Eviděncias. Parte II - Buscando as Eviděncias em Fontes de Informação. Revista Brasileira de Reumatologia, v. 44, n. 6, p. 403-409, dez. 2004.

BLAZEVICH, A.J.; GILL, N.D.; ZHOU, S. Intra- and intermuscular variation in human quadriceps femoris architecture assessed in vivo. Journal of Anatomy, v. 209, n. 3, p. 289-310, set. 2006.

BLAZEVICH, A.J.; SHARP, N.C. Craig. Understanding muscle architectural adaptation: Macro- and micro-level research. Cells Tissues Organs, v.181, n. 1, p.1-10, 2005.

CADORE, E.L. et al. Muscle conduction velocity, strength, neural activity, and morphological changes after eccentric and concentric training. Scandinavian Journal of Medicine and Science in Sports, v. 24, n. 5, p. e343-e352, fev. 2014.

CARPES, Felipe Pivetta; BINI, Rodrigo; VAZ, Marco Aurélio. Anatomia Funcional. São Paulo: Phorte; 2011.

CLARK, D.J. et al. Does quadriceps neuromuscular activation capability explain walking speed in older men and women? Experimental Gerontology, v. 55, p. 49-53, jul. 2014.

CORREA, C.S. et al. Effects of strength training, detraining and retraining in muscle strength, hypertrophy and functional tasks in older female adults. Clinical Physiology and Functional Imaging, v. 36, n. 4, p. 306-310, fev. 2016. 
CRUZ-JENTOFT, A.J. et al. Sarcopenia: European consensus on definition and diagnosis. Age and Ageing, v. 39, n. 4, p. 412-423, abr. 2010.

DIAS, C.P. et al. Effects of eccentric-focused and conventional resistance training on strength and functional capacity of older adults. Age, v. 37, n. 5, set. 2015.

FERRUCCI, L. et al. Age-related change in mobility: Perspectives from life course epidemiology and geroscience. The Journals of Gerontology Series A, v. 71, n. 9, p. 1184-1194, mar. 2016.

FRANCHI, M.V. et al. Architectural, functional and molecular responses to concentric and eccentric loading in human skeletal muscle. Acta Physiologica, v. 210, n. 3, p. 642-654, fev. 2014.

FRANCHI, M.V. et al. Early structural remodeling and deuterium oxide-derived protein metabolic responses to eccentric and concentric loading in human skeletal muscle. Physiological Reports, v.3, n. 11, p. e12593, nov. 2015.

FUKUNAGA, T. et al. Muscle architecture and function in humans. Journal of Biomechanics, v. $30, \mathrm{n}$. 5, p. 457-463, mai. 1997.

GANS, C; GAUNT, A.S. Muscle architecture in relation to function. Journal of Biomechanics, v. 24, p. 53-65, jan. 1991.

GUADAGNIN, E.C. et al. Correlation between lower limb isometric strength and muscle structure with normal and challenged gait performance in older adults. Gait and Posture, v. 73, set. 2019.

KAWAKAMI, Y; ABE, T; FUKUNAGA, T. Muscle-fiber pennation angles are greater in hypertrophied than in normal muscles. Journal of Applied Physiology, v. 74, n. 6, p. 2740-2744, jun. 1993.

KAWAKAMI, Y. The Effects of Strength Training on Muscle Architecture in Humans. International Journal of Sport and Health Science, v. 3, n. Special_Issue_2, 2005.

KOMFORTI, D. et al. Does skeletal muscle morphology or functional performance better explain variance in fast gait speed in older adults? Aging Clinical and Experimental Research, mai. 2020.

KUBO, K. et al. Muscle architectural characteristics in young and elderly men and women. International Journal of Sports Medicine, v. 24, n. 2, p. 125-130, fev. 2003.

KUMAGAI, K. et al. Sprint performance is related to muscle fascicle length in male 100-m sprinters. Journal of Applied Physiology, v. 88, n. 3, p. 811-816, mar. 2000.

LANDI, F. et al. Age-Related Variations of Muscle Mass, Strength, and Physical Performance in Community-Dwellers: Results From the Milan EXPO Survey. Journal of the American Medical Directors Association, v. 18, n. 1, p. 88.e17-88.e24, jan. 2017.

LASTAYO, P. et al. Eccentric versus traditional resistance exercise for older adult fallers in the community: A randomized trial within a multi-component fall reduction program. BMC Geriatrics, v. 17, n. 1, jul. 2017.

LEXELL, J.; TAYLOR, C.C.; SJÖSTRÖM, M.. What is the cause of the ageing atrophy?. Total number, 
size and proportion of different fiber types studied in whole vastus lateralis muscle from 15- to 83-yearold men. Journal of the Neurological Sciences, [S. 1.], v. 84, n. 2-3, p. 275-294, abr. 1988.

LIEBER, Richard L. Skeletal Muscle Structure and Function: Implications for Physical Therapy and Sports Medicine. Baltimore: Willians \& Wilkins, 1992.

LIEBER, R.L.; FRIDÉN, J. Functional and clinical significance of skeletal muscle architecture. Muscle Nerve v. 23, n. 11, p. 1647-1666, 2000.

LIPPERT, S. Lynn. Cinesiologia Clínica e Anatomia. 6ªed. Rio de Janeiro: Guanabara Koogan, 2018.

LIPPERT, S. Lynn. Cinesiologia clínica e anatomia aplicada. $5^{\text {a }}$ ed: Rio de Janeiro: Guanaba Koogan, 2011.

LOPEZ, P. et al. Echo intensity independently predicts functionality in sedentary older men. Muscle and Nerve, v. 55, n. 1, p. 9-15, out. 2017.

MATEOS-ANGUlO, A.; GALÁN-MERCANT, A..; CUESTA-VARGAS, A.I. Muscle thickness contribution to sit-to-stand ability in institutionalized older adults. Aging Clinical and Experimental Research, v. 32, n. 8, p. 1477-1483, ago. 2020.

MEN, Y. et al. The size and strength of the quadriceps muscles of old. Clinical Physiology, v. 5, n. 2, p. 145-154, abr. 1985.

MORSE, C.I. et al. Changes in triceps surae muscle architecture with sarcopenia. Acta Physiologica Scandinavica, v. 183, n. 3, p. 291-298, mar. 2005.

NARICI, M.V. et al. Effect of aging on human muscle architecture. Journal of Applied Physiology, v. 95, n. 6, p. 2229-2234, dez. 2003.

NARICI, M.V.; MAGANARIS, C.N. Adaptability of elderly human muscles and tendons to increased loading. Journal of Anatomy, v. 208, n. 4, p. 433-443, abr. 2006.

NEUMANN Donald A. Cinesiologia do aparelho musculoesquelético. $2^{\text {a }}$ ed. Rio de Janeiro: Elsevier, 2011.

NILWIK, R. et al. The decline in skeletal muscle mass with aging is mainly attributed to a reduction in type II muscle fiber size. Experimental Gerontology, v. 48, n. 5, p. 492-498, mai. 2013.

NORDIN Margareta; FRANKEL, Victor H. Biomecânica básica do sistema musculoesquelético. $4^{\mathrm{a}}$ ed. Rio de Janeiro: Guanabara Koogan, 2014.

NORONHA, DP; FERREIRA, SMSP. Revisões de literatura. In: CAMPELLO, B.S.V.C; CENDÓN, B.V; KREMER, J.M. (Org.). Fontes de informação para pesquisadores e profissionais. Belo Horizonte: UFMG, 2000.

PERKISAS, S. et al. Physiological and architectural changes in the ageing muscle and their relation to strength and function in sarcopenia. European Geriatric Medicine, v. 7, n. 3, p. 201-206, jun. 2016. 
RECH, A. et al. Echo intensity is negatively associated with functional capacity in older women. Age, v. 36, n. 5, ago. 2014.

REEVES, N.D. et al. Differential adaptations to eccentric versus conventional resistance training in older humans. Experimental Physiology, v. 94, n. 7, p. 825-833, jun. 2009.

SELVA RAJ, I.; BIRD, S.R.; SHIELD, A.J. Ultrasound Measurements of Skeletal Muscle Architecture Are Associated with Strength and Functional Capacity in Older Adults. Ultrasound in Medicine and Biology, v. 43, n. 3, p. 586-594, mar. 2017.

TIMMINS, R.G. et al. Architectural Changes of the Biceps Femoris Long Head after Concentric or Eccentric Training. Medicine and Science in Sports and Exercise, v. 48, n. 3, p. 499-508, mar. 2016.

VÁCZI, M. et al. Mechanical, hormonal, and hypertrophic adaptations to 10 weeks of eccentric and stretch-shortening cycle exercise training in old males. Experimental Gerontology, v. 58, p. 69-77, out. 2014.

WATANABE, Y. et al. Association between echo intensity and attenuation of skeletal muscle in young and older adults: A comparison between ultrasonography and computed tomography. Clinical Interventions in Aging, v. 13, p. 1871-1878, out. 2018.

WILHELM, E.N. et al. Relationship between quadriceps femoris echo intensity, muscle power, and functional capacity of older men. Age, v. 36, n. 3, p. 1113-1122, fev. 2014.

WISDOM, K.M.; DELP, S.L.; KUHL, E. Use it or lose it: multiscale skeletal muscle adaptation to mechanical stimuli. Biomechanics and Modeling in Mechanobiology, v. 14, n. 2, p. 195-215, set. 2015. WU, R. et al. Effects of age and sex on neuromuscular-mechanical determinants of muscle strength. Age, v. 38, n. 3, mai. 2016.

YOUNG, A.; STOKES, M.; CROWE, M. Size and strength of the quadriceps muscles of old and young women. European Journal of Clinical Investigation, v. 14, n. 4, p. 282-287, ago. 1984.

\section{Como citar este artigo}

POMPEO, K.D; GUADAGNIN, E.C; DOS SANTOS, P.F; DA ROCHA, E.S; RODRIGUES, R. Qual a influência da arquitetura muscular na funcionalidade de idosos? Uma revisão da literatura. Revista Kinesis, Santa Maria, v. 39, p.01-16, 2021.

\section{Agradecimentos}

Agradecemos ao Prof. Carlos de La Fuente pela revisão do espanhol.

* O presente manuscrito não contou com apoio financeiro de qualquer natureza. 